\title{
RESPONSABILIDADE SOCIAL CORPORATIVA E IDENTIFICAÇÃO ORGANIZACIONAL: INFLUÊNCIA NO ENGAJAMENTO DOS FUNCIONÁRIOS
}

\section{CORPORATE SOCIAL RESPONSIBILITY AND ORGANIZATIONAL IDENTIFICATION: INFLUENCE ON EMPLOYEE COMMITMENT}

\section{RESPONSABILIDAD SOCIAL CORPORATIVA E IDENTIFICACIÓN ORGANIZACIONAL: INFLUENCIA EN EL COMPROMISO DE LOS EMPLEADOS}

Recebido em: 15/02/2017

Avaliado em: 11/09/2018

Reformulado em:19/09/2018

Aceito para publicação em: 29/11/2018

Publicado em: 31/12/2020

Editor Responsável: Moacir M. Rodrigues Junior
Edicreia Andrade dos Santos ${ }^{1}$

Simone Soares ${ }^{2}$

Jonatas Dutra Sallaberry ${ }^{3}$

Carlos Alberto Marcal Gonzaga ${ }^{4}$

\section{RESUMO}

Este estudo teve por objetivo verificar em que medida o engajamento dos funcionários de um grupo de empresas é influenciado por suas percepções acerca das práticas de responsabilidade social corporativa (RSC), mediado pela identificação organizacional. Para alcançar o objetivo, foi utilizado um questionário com 189 respostas válidas, aplicado a funcionários de um grupo de empresas do Paraná. Para análise, utilizou-se Modelagem de Equações Estruturais processada pelo software SmartPLS. Os resultados indicam relação positiva e significativa entre o comprometimento dos funcionários e suas percepções das práticas de RSC. Também evidenciam a identificação organizacional como mediadora desta relação. Conclui-se que a percepção da RSC tem o maior impacto sobre os funcionários quando eles se sentem mais identificados com a organização. Este estudo também contribui para demonstrar a importância de investigar a RSC sob um nível individual de análise.

Palavras-chave: Responsabilidade Social Corporativa; Engajamento do funcionário. Identificação Organizacional; Empresa familiar.

\footnotetext{
${ }^{1}$ Doutora em Contabilidade pela Universidade Federal de Santa Catarina (UFSC); Professora da Universidade Federal do Paraná (UFPR); Email: edicreiaandrade@yahoo.com.br

${ }^{2}$ Mestranda em Desenvolvimento Comunitário pela Universidade Estadual do Centro-Oeste (UNICENTRO); Email: simosoares@gmail.com

${ }^{3}$ Doutorando em Contabilidade pela Universidade Federal de Santa Catarina (UFSC); E-mail: jonatas.sallaberry@hotmail.com

${ }^{4}$ Doutor em Engenharia Florestal pela Universidade Federal do Paraná (UFPR). Professor no Programa de Pós-Graduação Interdisciplinar em Desenvolvimento Comunitário (PPGDC) da UNICENTRO. E-mail: gonzaga@unicentro.br
} 


\begin{abstract}
This study aimed to verify to what extent the commitment of employees of a group of companies is influenced by their perceptions about the practices of corporate social responsibility (CSR), mediated by organizational identification. To achieve the objective, a questionnaire with 189 valid responses was used, applied to employees of a group of companies in Paraná. For analysis, Structural Equation Modeling was used, processed by the SmartPLS software. The results indicate a positive and significant relationship between the employees' commitment and their perceptions of CSR practices. They also show organizational identification as a mediator of this relationship. It is concluded that the perception of CSR has the greatest impact on employees when they feel more identified with the organization. This study also contributes to demonstrate the importance of investigating CSR from an individual level of analysis.
\end{abstract}

Keywords: Corporate Social Responsibility. Employee engagement. Organizational Identification. Family business.

\title{
RESUMEN
}

Este estudio tuvo como objetivo verificar en qué medida la participación de los empleados de un grupo de empresas está influenciada por sus percepciones sobre las prácticas de responsabilidad social corporativa (RSC), mediadas por la identificación organizacional. Para lograr el objetivo, se utilizó un cuestionario con 189 respuestas válidas, aplicado a los empleados de un grupo de empresas en Paraná. Para el análisis, se utilizó el modelado de ecuaciones estructurales, procesado por el software SmartPLS. Los resultados indican una relación positiva y significativa entre el compromiso de los empleados y sus percepciones de las prácticas de RSC. También muestran identificación organizacional como mediador de esta relación. Se concluye que la percepción de RSC tiene el mayor impacto en los empleados cuando se sienten más identificados con la organización. Este estudio también ayuda a demostrar la importancia de investigar la RSC desde un nivel de análisis individual.

Palabras-clave: Responsabilidad Social Corporativa. Compromiso de los empleados. Identificación organizacional. Empresa familiar.

\section{INTRODUÇÃO}

A Responsabilidade Social Corporativa (RSC) tornou-se um dos temas organizacionais mais importantes para a agenda do século XXI e teve um aumento considerável de pesquisas, principalmente, a partir dos anos 1990 (Azim, 2016). De acordo com Turker (2009), essa temática está relacionada diretamente com a criação de impactos positivos para as organizações e para os seus stakeholders. Em geral, a RSC é vinculada ao desempenho empresarial percebido pelos interessados (Carroll, 1991), pois o envolvimento da organização com questões de interesse social, fortalece sua relação com os stakeholders (Freguete, Nossa, \& Funchal, 2015).

As pesquisas de RSC tem enfatizado diversas abordagens, mas poucos estudos têm considerado os funcionários para o resultado do desempenho da RSC das organizações (Azim, 2016). Os funcionários podem sentir-se identificados e/ou engajados com as práticas de RSC da organização, o que refletirá em seu desempenho. Por exemplo, Albdour e Altarawneh (2012) realizaram um estudo acerca dos efeitos da RSC interna e o engajamento dos funcionários e concluíram que ela é uma boa preditora dos resultados do funcionário, do sucesso organizacional e do desempenho financeiro.

O engajamento do indivíduo no trabalho é positivo para ele e para a organização. Caracterizase por uma elevada identificação do indivíduo com a sua atividade e com a organização, que resultam em bem-estar e satisfação (Bakker \& Leiter, 2010). Bakker (2011) evidenciou que os funcionários engajados na realização de suas atividades funcionais são cognitivamente, emocionalmente e fisicamente conectados com seus papéis na função laboral. Este mesmo autor também constatou que 
o engajamento é um importante sinal de bem-estar ocupacional e, por isso, os gestores devem fomentar o envolvimento e a identificação de seus funcionários com a organização.

A identificação organizacional é uma das formas de identificação social, e ocorre quando o indivíduo se define como integrante de uma organização (Ashfort \& Mael, 1989; Mael \& Ashforth, 1992; Dutton, Dukerith \& Harquail, 1994). O indivíduo sente-se identificado ao incorporar os valores, crenças e atributos da organização na qual trabalha em seu autoconceito. Essa identificação do indivíduo possui forte natureza cognitiva, distinguindo-se de outros atributos associáveis a comportamentos ou posicionamentos afetivos, dentre os quais lealdade e dedicação.

Sob o aspecto social, uma organização é percebida como detentora de um conjunto de características e/ou atributos que seus integrantes internalizam como seus, gerando importante vínculo entre ela e seus funcionários (Marra, Fonseca, \& Marques, 2014). Uma maior identificação com a organização resulta em comportamentos mais alinhados com suas crenças, normas e valores (Ashforth \& Mael, 1989; Mael \& Ashforth, 1992). Isso explica a importância deste construto para entender a percepção dos funcionários com relação aos projetos de RSC promovidos pela empresa em que trabalham.

Com base no exposto, este estudo almeja responder a seguinte questão: em que medida a Responsabilidade Social Corporativa mediada pela identificação organizacional influencia no engajamento dos funcionários? Assim, o estudo objetiva analisar em que medida o engajamento dos funcionários de uma organização é influenciado pelas percepções das práticas de RSC, mediado pela identificação organizacional. Para conduzir tal investigação, foi realizado um estudo de caso com os funcionários de um grupo de empresas familiares localizadas na região centro sul do estado do Paraná.

Há poucos estudos empíricos ou teóricos sobre o desenvolvimento do engajamento dos funcionários e dos fatores que o incentivam ou o inibem (Saks, 2006; Ferreira \& Oliveira, 2014). Os antecedentes e os resultados do engajamento dos funcionários são conceituados em apenas alguns raros modelos (Shuck \& Wollard, 2010; Ferreira \& Oliveira, 2014). Dentre as poucas pesquisas predominam as abordagens em nível macro ou institucional (Aguinis \& Glavas, 2013; Glavas, 2016; Lee, 2008; Wood, 2010), ou focadas em nos impactos externos da organização (Hansen, Dunford, Boss, Boss \& Angermeier, 2011).

De acordo com Glavas e Piderit (2009) e Glavas (2016), a percepção positiva dos funcionários acerca da RSC praticada em suas organizações afeta seu engajamento com a organização e com o trabalho, porém as pesquisas ainda são incipientes. Glavas (2016) sustenta a ideia de que a relação positiva entre RSC e engajamento dos indivíduos se assenta na percepção que as organizações atuam conforme os princípios de suas declarações. Isso reforça, os valores organizacionais para os funcionários, impulsionando o aumento da sua identificação com a organização (Glavas, 2016; Peterson, 2004).

Este artigo apresenta o resultado da pesquisa realizada. Está estruturado em cinco seções, das quais esta introdução é a primeira. A segunda seção é composta por uma revisão bibliográfica da literatura publicada relacionada ao tema focado, com destaque para as abordagens sobre Responsabilidade Social Corporativa, engajamento do funcionário e identificação organizacional. A terceira seção apresenta a metodologia e explana os detalhes dos procedimentos adotados. A quarta seção expõe os resultados e sua discussão. Para concluir os autores elaboram, na quinta seção, suas considerações finais, comentando sobre as limitações da pesquisa e possibilidades de pesquisas futuras.

\section{REFERENCIAL TEÓRICO}

\subsection{Responsabilidade Social Corporativa (RSC)}

A RSC é caracterizada como um conjunto de obrigações que os tomadores de decisões seguem para alinhar suas entidades aos valores e fins da sociedade (Bowen, 1957). Na década de 1970, a RSC ganhou relevância nas discussões de problemas sociais e academicamente teve grandes contribuições 
de Carroll (1979), as quais dizem respeito às expectativas éticas, sociais, econômicas e legais das organizações para com a sociedade. Neste aspecto, as organizações estariam acompanhando interesses sociais determinados pelo contexto econômico ou pelo próprio mercado, embora algumas necessidades da comunidade não se alteram com o tempo (Carroll, 1979).

A RSC é pesquisada por diferentes vertentes e disciplinas, com foco tanto nos aspectos internos das organizações, quanto nos externos (Ferreira \& Oliveira, 2014). De acordo com Turker (2009), o desenvolvimento de ações de responsabilidade social, externas ou internas, aumentam o comprometimento dos funcionários. Em alguns casos contribui para melhorar o clima organizacional e o desejo de permanecer trabalhando na organização.

As práticas internas de RSC estão relacionadas ao ambiente de trabalho físico e psicológico dos funcionários (Turker, 2009). Expressa-se na preocupação com a saúde e o bem-estar laboral, focando em treinamento, participação nos negócios, igualdade de oportunidades, relacionamento familiar e similares (Ferreira \& Oliveira, 2014). As práticas externas de RSC referem-se a ações direcionadas à comunidade local, parceiros de negócios, fornecedores, clientes, autoridades públicas, ONGs e outros stakeholders (Ferreira \& Oliveira, 2014).

Uma parte da literatura sobre RSC concentra-se nas responsabilidades corporativas para com os grupos de stakeholders (Ferreira \& Oliveira, 2014; Serpa \& Fourneau, 2007), definidos por Freeman (2010) como qualquer grupo ou indivíduo que pode afetar ou ser afetado por seus objetivos e práticas. Eles influenciam e são influenciados pelas decisões da organização, impactando em sua sobrevivência. Por isso, os gestores precisam aprender a desenvolver metas que os satisfaçam (Freeman, 2010).

Os funcionários formam um dos grupos de stakeholders mais envolvido na implementação organizacional de comportamentos responsável (Collier \& Esteban, 2007). Por isso, as práticas de RSC dependem, em grande parte, do comprometimento deles para serem desempenhadas. A identificação com a postura socialmente responsável da organização, ao estabelecer políticas de RSC podem exercer um efeito positivo no comprometimento dos funcionários (Peterson, 2004). Rios (2011) enfatiza que os investimentos no capital humano fazem parte de uma estratégia corporativa eficaz para acompanhar as responsabilidades impostas pelo mercado, incentivando estudos acerca do comportamento organizacional e dos indivíduos, bem como de assuntos relacionados, como satisfação, vínculos com o trabalho, engajamento, e outros indicadores, como o tempo de serviço etc.

De acordo com Hansen, Dunford, Boss, Boss e Angermeier (2011) pesquisas sobre RSC tendem a se concentrar em stakeholders e resultados externos, em vez de focar nos aspectos internos que relacionam a RSC com o desempenho da empresa e seu impacto nos stakeholders internos, como funcionários. Por isso alguns estudos foram realizados sobre a relação das práticas de RSC e seu relacionamento com os funcionários, aumentando, por exemplo, a identificação e o comprometimento com a organização, os comportamentos de cidadania organizacional, engajamento do funcionário e a significância do trabalho, entre outras atitudes e comportamentos (Ferreira \& Oliveira, 2014; Glavas \& Godwin, 2013; Peterson, 2004). Outros estudos referem que a RSC também pode melhorar a capacidade das empresas de atrair e manter os melhores talentos (Bhattacharya, Sen, \& Korschun, 2008). Por isso, é importante ainda que sejam desenvolvidas as pesquisas envolvendo a RSC e variáveis sentimentais, atitudinais, comportamentais dos indivíduos como engajamento e identificação organizacional.

\subsection{Engajamento do Funcionário}

O engajamento do funcionário é entendido como uma condição desejável que favorece o desempenho individual e organizacional (Oliveira \& Rocha, 2017), os quais são gerados por comprometimento e alinhamento a partir de objetivos comuns (Bakker, Albrecht, \& Leiter, 2011). A literatura apresenta várias conclusões e afirmações acerca do engajamento de trabalhadores numa organização. Kahn (1990) e Tsourvakas e Yfantidou (2018) enfatizam a conexão física, cognitiva e emocional dos indivíduos com as atividades que desempenham no trabalho. Ferreira e Oliveira (2014) 
destacam a maior disposição dos indivíduos para contribuírem com as metas organizacionais, sendo mais produtivos e menos propensos ao estresse e absenteísmo. Tsourvakas e Yfantidou (2018) afirmam que um funcionário engajado se familiariza com a perspectiva de negócios e melhora o desempenho em equipe. Rich, Lepine e Crawford (2010) concluem que o esforço das organizações em desenvolver práticas de RSC que incentivam a identificação organizacional e o engajamento funcional deve-se ao seu reflexo no aumento do desempenho no trabalho.

O engajamento é considerado resultado de um relacionamento de mão dupla dentro das organizações, entre o empregador e o empregado, entre o nível estratégico e o operacional (Robinson, Hooker, \& Hayday, 2007). Os fatores motivacionais para o engajamento dos funcionários são enumerados por Robinson et al. (2007) e Tsourvakas e Yfantidou (2018), incluindo as avaliações, a comunicação eficiente, o plano de desenvolvimento de carreira, os bônus, as oportunidades de equilíbrio entre vida pessoal e profissional, as condições de trabalho, a satisfação com o tipo de trabalho, as relações com a gerência imediata, trabalho em equipe e outras variáveis do ambiente organizacional.

Ferreira e Oliveira (2014) elaboraram uma diferenciação conceitual entre engajamento, comprometimento e envolvimento, recusando o uso intercambiável das expressões. Eles ressaltam que o engajamento se refere à identificação cognitiva e psicológica do trabalho, incluindo a ideia de que ele satisfaz necessidades e expectativas. O comprometimento refere-se aos aspectos atitudinais e afetivos de vínculo emocional, com base em valores e interesses compartilhados com a organização.

Ferreira e Oliveira (2014) analisaram a relação entre RSC e o engajamento dos funcionários, com aplicação de um experimento com três diferentes cenários de RSC (geral, interna, externa). Os resultados mostraram que não há diferenças estatisticamente significativas nos níveis de engajamento entre funcionários expostos a práticas externas e internas de RSC. No entanto, os funcionários expostos à RSC interna estão mais engajados do que aqueles expostos apenas a práticas externas. Os autores recomendaram que outros estudos fossem realizados por meio de outros procedimentos metodológicos, e que as empresas analisassem suas estratégias de RSC de uma maneira holística, ou seja, interna e externa.

Yousaf, Ali, Sajjad e Ilyas (2016) buscaram analisar o impacto da RSC interna no engajamento dos funcionários por meio da mediação da percepção e influência da discrição dos funcionários. Os resultados mostraram que a RSC interna tem impacto direto e indireto na percepção dos funcionários e sobre o seu engajamento. Confirmaram que um efeito indireto da percepção da RSC interna no engajamento dos funcionários depende do nível de discrição no ambiente de trabalho.

Os níveis de engajamento dos funcionários e seus resultados podem ser decorrentes do desempenho da RSC na organização (Albdour \& Altarawneh, 2012; Azim, 2016). Com base no exposto, apresenta-se a seguinte hipótese:

H1: Existe uma relação positiva entre a RSC percebida da empresa e o engajamento dos funcionários.

\subsection{Identificação Organizacional}

A identificação organizacional é derivada da Teoria da Identidade Social (TIS) (Ashforth \& Mael 1989; Tajfel \& Turner 1979) e refere-se a uma ligação cognitiva entre a definição da organização e a definição do eu (Dutton, Dukerich, \& Harquail, 1994). Isso faz com que o indivíduo perceba como fossem seus os sucessos e fracassos da organização (Mael \& Ashforth 1992). De acordo com a TIS, é por meio da identificação que os indivíduos se definem como membros de categorias sociais que os ajudam a se diferenciar uns dos outros e a entender os aspectos que compartilham com os demais membros de seus grupos de pertencimento (Ashfort \& Mael, 1989; Glavas \& Godwin, 2013). Portanto, a identificação organizacional é uma das formas mais importantes de ligação dos empregados à organização. 
A identificação organizacional tem sido percebida como uma importante variável para o entendimento da identidade dos indivíduos e a explicação de suas atitudes e comportamentos no ambiente das organizações (Tavares, 2001). Jenkins (2005) destaca que ao se identificar, o indivíduo absorve as crenças da organização em sua própria identidade, seu autoconceito incorpora características que para ele definem a organização como um grupo social, passando a compor aquilo que é, ou seja, fazendo parte de seu próprio eu.

Supondo que a identificação organizacional está diretamente relacionada ao engajamento dos funcionários, à satisfação no trabalho, à lealdade organizacional, e ao envolvimento com o trabalho, conjectura-se a seguinte hipótese:

\section{H2: Existe uma relação positiva entre identificação organizacional e o engajamento do funcionário.}

Verificou-se que ocorre aumento da identificação e do comprometimento com a organização, dos comportamentos de cidadania organizacional, do engajamento e da significância do trabalho (Ferreira \& Oliveira, 2014; Glavas \& Godwin, 2013; Peterson, 2004). Pratt (1998) conjectura que funcionários de organizações associadas a uma visão ou causa social específica podem ter aumentado a identificação organizacional porque se sentem mais conectados à organização do que a afiliação típica poderia prever. Da mesma forma, as pesquisas de Dutton, Roberts e Bednar (2010) e de Glavas e Godwin (2013) apontam que os funcionários estão mais propensos a se identificarem positivamente com empresas nas quais percebem boas virtudes, força de caráter e responsabilidade social.

Outras pesquisas observaram que um alto nível de RSC é mais importante, para alguns indivíduos, do que fatores como altos salários (Glavas \& Godwin, 2013; Montgomery \& Ramus, 2008). Joyner e Payne (2002) identificaram, num estudo nos EUA, que $86 \%$ dos funcionários que consideravam suas organizações socialmente responsáveis sentiam-se fortemente identificados com a organização, enquanto apenas $14 \%$ daqueles que consideravam sua organização como baixa em RSC se sentiam identificados a ela. A partir dessas constatações, conjectura-se que a identificação organizacional pode ser um elemento mediador entre a percepção das práticas de RSC que o funcionário tem de sua organização e o seu engajamento. Assim propõe-se a seguinte hipótese:

H3: A identificação organizacional atua como mediadora no aumento do engajamento do funcionário ao perceber maior RSC em sua organização.

Com base no descrito e nas hipóteses destacadas, evidencia-se na Figura 1 o modelo teórico proposto para esta pesquisa.

Figura 1. Modelo Conceitual da Pesquisa

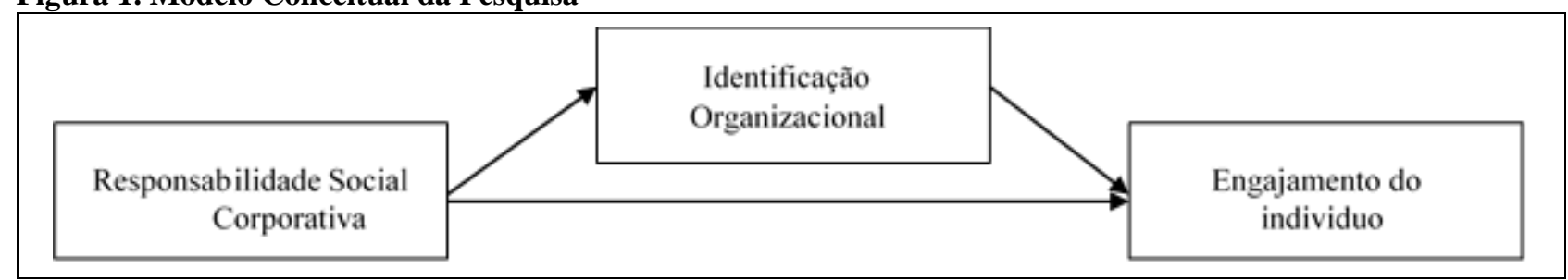

Fonte: Elaborado pelos autores.

A síntese das hipóteses desta pesquisa é de que quanto mais positivamente a prática da RSC de uma organização for percebida pelos funcionários, maior será o seu grau de engajamento e identificação organizacional. Dialeticamente, quanto maior for o grau de engajamento, mais positiva tende a ser a percepção da RSC mediada pela identificação organizacional. 


\section{PROCEDIMENTOS METODOLÓGICOS}

\subsection{Amostra e Instrumento de pesquisa}

Tabela 1. Construtos de Pesquisa

\begin{tabular}{|c|c|c|}
\hline Construto & Variáveis & Assertivas \\
\hline \multirow{3}{*}{$\begin{array}{c}\text { RSC } \\
\text { (Turker, 2009) }\end{array}$} & $\begin{array}{c}\text { RSC para } \\
\text { partes } \\
\text { interessadas } \\
\text { sociais e não } \\
\text { sociais } \\
\text { (RSC.PI) }\end{array}$ & $\begin{array}{l}\text { 1. A minha empresa apoia as organizações não governamentais que trabalham nas áreas } \\
\text { problemáticas (Ex. ANAPCI). } \\
\text { 2. A minha empresa participa nas atividades que visam proteger e melhorar a qualidade } \\
\text { da comunidade na qual está inserida. } \\
\text { 3. A minha empresa implementa programas especiais para minimizar seu impacto } \\
\text { negativo no meio ambiente (descarte correto dos lixos, papelóes, etc.). } \\
\text { 4. A minha empresa contribui para as campanhas e projetos que promovem o bem-estar } \\
\text { da sociedade (Ex.: X solidário). }\end{array}$ \\
\hline & $\begin{array}{l}\text { RSC para os } \\
\text { funcionários } \\
\text { (RSC.FUNC) }\end{array}$ & $\begin{array}{l}\text { 1. A minha empresa incentiva seus funcionários a participarem voluntariamente das } \\
\text { atividades sociais. } \\
\text { 2. As políticas da nossa empresa incentivam os funcionários a desenvolver suas } \\
\text { habilidades e carreiras. } \\
\text { 3. A minha empresa implementa políticas flexíveis para proporcionar um bom } \\
\text { equilíbrio entre vida e trabalho a seus funcionários. } \\
\text { 4. As decisões gerenciais relacionadas com os funcionários são geralmente justas. } \\
\text { 5. A minha empresa apoia funcionários que desejam adquirir educação adicional. }\end{array}$ \\
\hline & $\begin{array}{l}\text { RSC para } \\
\text { clientes } \\
\text { (RSC.C) }\end{array}$ & $\begin{array}{l}\text { 1. A minha empresa protege os direitos do consumidor além dos requisitos legais. } \\
\text { 2. A minha empresa fornece informações completas e precisas sobre seus produtos para } \\
\text { seus clientes. } \\
\text { 3. A satisfação do cliente é muito importante para a minha empresa. }\end{array}$ \\
\hline $\begin{array}{l}\text { Engajamento } \\
\text { Individual (EI) } \\
\text { (Tsourvakas \& } \\
\text { Yfantidou, } \\
\text { 2018) }\end{array}$ & EI & $\begin{array}{l}\text { 1. Eu falo muito bem desta empresa para meus amigos. } \\
\text { 2. Ficaria feliz por minha família e meus amigos usarem os produtos ou serviços desta } \\
\text { empresa. } \\
\text { 3. Esta empresa é conhecida na comunidade como um bom empregador. } \\
\text { 4. Esta empresa tem uma boa reputação. } \\
\text { 5. Tenho orgulho de dizer aos outros que faço parte desta empresa. } \\
\text { 6. Esta empresa realmente inspirou o que há de melhor em mim no desempenho do } \\
\text { meu trabalho. } \\
\text { 7. Eu acho que meus valores e os da empresa são muito semelhantes. } \\
\text { 8. Eu sempre faço mais que é realmente necessário. } \\
\text { 9. Eu tento ajudar os outros colegas nesta empresa sempre que posso. } \\
\text { 10. Eu tento me manter a par dos novos conhecimentos na minha área/função sempre } \\
\text { que posso. } \\
\text { 11. Sou voluntário para coisas fora do meu trabalho que contribuem para os objetivos } \\
\text { da empresa. } \\
\text { 12. Eu frequentemente faço sugestões para melhorar o trabalho da minha equipe/setor. }\end{array}$ \\
\hline $\begin{array}{c}\text { Identificação } \\
\text { Organizacional } \\
\text { (IO) } \\
\text { (Mael \& } \\
\text { Ashforth, } \\
\text { 1992) }\end{array}$ & IO & $\begin{array}{l}\text { 1. Quando alguém critica a empresa, sinto isso como um insulto pessoal. } \\
\text { 2. Eu estou verdadeiramente interessado no que os outros pensam acerca desta empresa. } \\
\text { 3. Quando falo desta empresa, digo mais vezes "nós" do que "eles". } \\
\text { 4. O sucesso desta empresa é o meu sucesso. } \\
\text { 5. Quando alguém elogia a empresa, sinto isso como um elogio pessoal. } \\
\text { 6. Se uma história nos meios de comunicação social criticasse a empresa sentir-me-ia } \\
\text { mal. }\end{array}$ \\
\hline
\end{tabular}

Fonte: Elaborado pelos autores.

A população desta pesquisa é constituída por 442 funcionários de um grupo de empresas geridas por um núcleo familiar, com várias atividades sociais em benefício de asilos, escolas e enfermos desenvolvidas na comunidade local. A organização foi escolhida para o estudo de caso devido a sua acessibilidade para coleta de dados. Do universo de funcionários, 289 responderam o 
questionário. Todavia, apenas 189 foram validados para a tabulação dos dados, com base no critério de que os funcionários deveriam ter no mínimo 6 meses de empresa, (critério da socialização com a cultura e valores organizacionais), pelo qual se excluiu 55 questionários, e porque o preenchimento de 45 deles estavam incompletos.

Para validar o tamanho da amostra necessária e o poder estatístico, utilizou-se da aplicação do software $\mathrm{G}^{*}$ Power. Para tanto, considerou-se o efeito total de 2 variáveis preditoras (RSC e identificação organizacional) sobre a variável dependente (engajamento do indivíduo), ao nível de significância de $5 \%$, e um poder estatístico de 0,95 . O cálculo indicou uma amostra mínima superior a 90 respostas válidas, de modo que a amostra de 189 respostas cumpre este requisito.

O questionário utilizado como instrumento de pesquisa foi aplicado presencialmente aos funcionários por um dos membros da equipe de pesquisa, no período de 7 a 15 de janeiro de 2019 . O questionário foi composto de três construtos: (i) RSC com três variáveis para entender as partes interessadas sociais e não sociais (RSC.PI), os funcionários (RSC.FUNC) e os clientes (RSC.C); (ii) Engajamento individual com 12 assertivas; e (iii) identificação organizacional (IO) com seis assertivas. Também foram listadas oito assertivas de dados demográficos para levantamento do perfil dos respondentes.

A escolha do instrumento de pesquisa deu-se por alinhamento ao referencial teórico de Carroll (1979), visto que tais construtos estariam mais alinhados à proposta de pesquisa de RSC. Turker (2009) desenvolveu a escala de forma independente, segregada em fatores no interesse da pesquisa, com elevadas cargas de comunalidades. Tsourvakas e Yfantidou (2018) adicionalmente exploram a literatura de engajamento para proporcionar itens de pesquisa contemporâneos nas relações entre engajamento e RSC, enquanto Mael e Ashforth (1992) reformularam e validaram os itens de identificação organizacional predominantes nas pesquisas sobre o tema.

Para resposta do questionário, exceto dos itens de dados demográficos, foi solicitado aos respondentes a indicação do seu grau de concordância com as assertivas que compunham as questões, utilizando-se uma escala tipo Likert, com cinco gradações: de concordo totalmente (1) a discordo totalmente (5). Antes da aplicação do instrumento de pesquisa realizaram-se os procedimentos de back-translation (Brislin, 1970) com dois profissionais habilitados e um pré-teste com vistas à validação externa (Yin, 1994). O pré-teste foi realizado com um sócio proprietário da empresa e três funcionários que atuam respectivamente como gerente de loja, encarregado de sessão e fiscal de frente de caixa. A partir do pré-teste algumas questões do instrumento inicial foram excluídas e outras questões tiveram suas redações adequadas conforme o contexto da organização.

\subsection{Análise dos Dados}

A análise dos dados foi realizada a partir da técnica de modelagem de equações estruturais (SEM) estimada a partir dos Mínimos Quadrados Parciais (Partial Least Squares - PLS). A técnica possibilita estimar uma série de equações de regressão múltiplas separadas, mas interdependentes, de forma simultânea, pela especificação do modelo estrutural (Dijkstra, 2010; Hair Jr., Hult, Ringle, \& Sarsted, 2016; Ringle, Wendw \& Becker, 2015).

Para a análise, realizou-se a avaliação do modelo de mensuração e do modelo estrutural. Também se realizou a verificação da mediação, a qual é entendida como mecanismo em que a variável independente influencia a variável dependente, por meio da transmissão de efeitos da variável mediadora (Baron \& Kenny, 1986). As validações decorreram de análises no bootstrapping (PLS) que consistem na melhor alternativa para se testar as hipóteses de mediação e fornecem um teste rigoroso da importância dos efeitos indiretos, avaliando e comparando os efeitos indiretos em modelos de mediação (Mackinon, Lockinnon, \& Williams, 2004). 


\section{RESULTADOS E DISCUSSÕES}

\subsection{Perfil dos Respondentes}

A análise descritiva dos dados permite conhecer as características da amostra da pesquisa. Assim, apresenta-se na Tabela 2 os dados demográficos dos 189 respondentes.

Tabela 2. Dados dos Respondentes

\begin{tabular}{lcc|lcc}
\hline Gênero & $\mathbf{N}$ & $\mathbf{( \% )}$ & Idade (Faixa etária) & $\mathbf{N}$ & $(\boldsymbol{\%})$ \\
\hline Feminino & 96 & $50,80 \%$ & De 18 a 21 anos & 40 & $21,40 \%$ \\
Masculino & 93 & $49,20 \%$ & De 22 a 30 anos & 80 & $42,80 \%$ \\
& & & Acima de 30 anos & 69 & $35,80 \%$ \\
Total & $\mathbf{1 8 9}$ & $\mathbf{1 0 0 \%}$ & Total & $\mathbf{1 8 9}$ & $\mathbf{1 0 0 \%}$ \\
\hline Escolaridade & $\mathbf{N}$ & $\mathbf{( \% )}$ & Tempo de Empresa & $\mathbf{N}$ & $\mathbf{( \% )}$ \\
\hline Ensino Fundamental Incompleto & 5 & $2,70 \%$ & De 6 meses a 3 anos & 73 & $38,80 \%$ \\
Ensino Fundamental & 31 & $16,50 \%$ & De 3 anos a 7 anos & 67 & $35,60 \%$ \\
Ensino Médio & 130 & $69,10 \%$ & Acima de 7 anos & 49 & $25,60 \%$ \\
Ensino Superior & 23 & $11,70 \%$ & & $\mathbf{1 8 9}$ & $\mathbf{1 0 0 \%}$ \\
Total & $\mathbf{1 8 9}$ & $\mathbf{1 0 0 \%}$ & Total & &
\end{tabular}

Fonte: Dados da pesquisa.

Observa-se a partir dos dados demográficos que a amostra é equilibrada quanto ao gênero sendo 50,8\% dos respondentes do gênero feminino (n: 96) e 49,2\% do gênero masculino (n: 93). Predominam colaboradores jovens, sendo que até 30 anos estão 64,2\% dos respondentes, com formação em sua maioria (88,3\% da amostra) até o nível de ensino médio completo. Somente $11,7 \%$ dos colaboradores alcançaram o ensino de nível superior completo.

A experiência dos respondentes nas empresas é mediana, haja visto que como critério da amostragem foram desconsiderados colaboradores com até seis meses, restando $38,8 \%$ na faixa de até 3 anos de experiência. $\mathrm{O}$ restante, com experiência no profissional no grupo superior a 3 anos é de $61,1 \%$ da amostra.

\subsection{Equações estruturais}

A análise dos dados com o emprego da técnica estatística de equações estruturadas, foi realizada por meio do software SmartPLS3.2.8. Para a avaliação da validade dos construtos, foram observadas a validade discriminante e a validade convergente. A validade discriminante foi a primeira a ser verificada, sendo que dos 30 indicadores iniciais constantes do instrumento de pesquisa, todos demonstraram-se satisfatórios, com a maior carga no construto indicado. No entanto, decorrente da análise da validade convergente, percebeu-se alguns construtos com cargas fatoriais menores do que o previsto, e com isso algumas variáveis precisaram ser excluídas, resultando no emprego de 21 variáveis observáveis restantes. As variáveis excluídas foram engajamento do funcionário (EF8, EF9, EF10, EF11, EF12) e RSC (RSC.C2, RSC.C3, RSC.FUNC1, e RSC.PI4).

Os coeficientes da validade convergente, da Confiabilidade Composta (CC), do Alfa de Cronbach apresentaram valores satisfatórios, respectivamente a partir de 0,5, 0,7 e 0,7 (Fornell \& Larcker, 1981), evidenciados na Tabela 3. Da mesma forma também é apresentado o valor de $\mathrm{R}^{2}$ para enriquecer as análises, sendo que não possui ponto de corte, embora o desejado seja o maior possível.

Tabela 3. Validades do Modelo

\begin{tabular}{|c|c|c|c|c|c|c|c|}
\hline \multirow{2}{*}{ Construtos } & \multirow{2}{*}{$\begin{array}{c}\text { Alfa de } \\
\text { Cronbach }\end{array}$} & \multirow{2}{*}{$\begin{array}{c}\begin{array}{c}\text { Confiabilidade } \\
\text { composta }\end{array} \\
\end{array}$} & \multirow{2}{*}{ AVE } & \multirow{2}{*}{$\mathbf{R}^{2}$} & \multicolumn{3}{|c|}{ Fornell-Larcker } \\
\hline & & & & & EI & IO & RSC \\
\hline EI & 0,903 & 0,924 & 0,636 & 0,720 & 0,798 & & \\
\hline $\mathrm{IO}$ & 0,861 & 0,896 & 0,590 & 0,334 & 0,730 & 0,768 & \\
\hline $\mathrm{RSC}$ & 0,858 & 0,890 & 0,504 & - & 0,774 & 0,578 & 0,780 \\
\hline
\end{tabular}

Fonte: Dados da pesquisa. 
A análise da Tabela 3 evidencia que todas as cargas para a AVE (Average Variance Extracted) foram estatisticamente significativas, isto é, com valores iguais ou superiores a 0,5 (Fornell \& Larcker, 1981). O indicador da Confiabilidade Composta atendeu aos valores limítrofes considerados satisfatórios da mesma forma que os valores do Alfa de Cronbach todos acima de 0,7 para as variáveis latentes.

A presença de indicadores satisfatórios de Confiabilidade Composta e Alfa de Cronbach sinalizam que a amostra está teoricamente livre de vieses e que o instrumento de coleta de dados aplicado é confiável (Hair Jr. et al., 2016). A apuração do alfa de Cronbach é empregada para verificar a consistência interna dos resultados de cada variável latente, que estabelece a expectativa de erro da medida feita, e quanto mais próximo de 1 , menor a expectativa de erro e maior a confiabilidade do instrumento (Hair Jr. et al., 2016). Nesta amostra, os resultados do Alfa de Cronbach apresentaram um grau de fidelidade aceitável.

Muito importante para a análise dos resultados, revelando sua relevância, é a identificação e apropriação dos coeficientes de determinação (R2) para as variáveis, que indica o quanto o modelo explica determinada variável. Neste estudo, foi demonstrado que o modelo explica $72 \%$ da variação do engajamento do indivíduo e 33\% da variação da identificação organizacional.

O passo seguinte de análise da validade discriminante é a verificação das variáveis latentes do modelo, que de acordo com Fornell e Larcker (1981), é confirmada quando o valor da raiz quadrada da AVE é maior do que os valores absolutos das correlações com as demais variáveis latentes. Ou seja, a diagonal principal precisa apresentar valores superiores ao das demais variáveis latentes, sendo que nesse teste, decorrente de valores insatisfatórios foi necessário realizar a exclusão das nove variáveis observáveis do modelo.

Na sequência realizou-se a análise do modelo estrutural que permite validar estatisticamente as relações entre os construtos e as conexões construídas segundo estrutura de um diagrama de caminhos em base teórica (Hair Jr. et al., 2016). Para avaliar a validade do modelo estrutural são analisados os critérios de (i) o tamanho e significância dos coeficientes de caminho e (ii) coeficientes de determinação de Pearson $\left(\mathrm{R}^{2}\right)$ por meio da técnica de Bootstrapping, e (iii) a Relevância Preditiva $\left(\mathrm{Q}^{2}\right)$ na plataforma blindfolding, e (iv) tamanhos do efeito $\left(\mathrm{F}^{2}\right)$ (Hair Jr. et al., 2016).

No bootstrapping, as subamostras são criadas com observações retiradas aleatoriamente do conjunto original de dados, para serem usadas na estimativa do modelo de caminhos do PLS. Neste caso, foram geradas cinco mil subamostras diferentes, conforme recomendado por Hair Jr et al. (2016). Tais resultados são apresentados na Tabela 4.

O valor de $Q^{2}$ do Stone-Geisser é um critério de relevância preditiva (Geisser, 1974; Stone, 1974). O cálculo de $Q^{2}$ das variáveis latentes é obtido por meio do procedimento blindfolding. Segundo Ringle, Wende e Becker (2015), o Blindfolding é uma técnica de reutilização da amostra, que sistematicamente exclui pontos de dados e fornece um prognóstico dos seus valores originais, o que exige a atribuição de uma distância de omissão "D" ( $\mathrm{D}=8)$.

A técnica preconiza que no PLS-SEM quando há relevância preditiva, prediz adequadamente os valores dos indicadores. Quando o valor de $\mathrm{Q}^{2}$ maior que zero para a variável latente endógena, significa que o modelo tem relevância preditiva para esse constructo. Nesta pesquisa os valores foram validados haja vista que para ambas as variáveis o resultado superou o zero.

Tabela 4. Efeitos Entre Construtos

\begin{tabular}{lccc}
\multicolumn{1}{c}{ Relação Estrutural } & Coef. & p-valor & Hipótese \\
\hline RSC -> Engajamento dos Funcionários & 0,529 & $0,000 * * *$ & H1 \\
Identificação Organizacional -> Engajamento dos Funcionários & 0,424 & $0,000^{* * *}$ & H2 \\
RSC -> Identificação Organizacional -> Engajamento do Funcionário & 0,578 & $0,000 * * *$ & H3 \\
\hline
\end{tabular}

Nota: $\mathrm{N}=189$. *** sig a $1 \% . \mathrm{Q}^{2}=$ Identificação Organizacional $(0,416)$; Engajamento dos Funcionários $(0,176)$.

$\mathrm{F}^{2}=$ Identificação Organizacional $(0,665)$; Engajamento dos Funcionários $(0,503)$.

Fonte: Dados da pesquisa 
Os resultados apresentados na Tabela 4 demonstram, estatisticamente, a confirmação das três hipóteses de pesquisa. Esta conclusão é verificável porque os valores da coluna do p-valor evidenciam que todas as relações estruturais foram significativas a $1 \%$. Os valores do coeficiente beta que representam os efeitos diretos entre as variáveis (H1: RSC e engajamento do funcionário; H2: identificação organizacional e engajamento dos funcionários), e efeitos indiretos (H3: RSC, identificação organizacional e engajamento do funcionário), confirmam as relações positivas entre as relações testadas. Por isso, é possível concluir que na empresa estudada ocorre uma influência positiva da percepção das práticas de RSC na identificação dos funcionários com a organização e, consequentemente, moldando o seu engajamento ao ambiente organizacional. Esses resultados corroboram outros estudos mencionados na revisão da literatura conforme são discutidos no tópico seguinte.

\subsection{Resultados}

Os resultados obtidos confirmam a ocorrência de identificação organizacional (42\%), de engajamento dos funcionários (53\%), e verifica-se $72 \%$ de explicação geral pelos construtos do estudo, tendo por referência os coeficientes de determinação de Pearson $\left(\mathrm{R}^{2}\right)$, o que é considerado como um grande efeito (Hair Jr. et al., 2016). Em parte, a constância das práticas de RSC pela organização estudada pode justificar essa variação, porque os funcionários se percebem inseridos na atuação da organização, como sendo sujeitos ativos e corresponsáveis pelas ações desenvolvidas.

Considerando os valores para $\mathrm{f}^{2}$ sugeridos por Hair Jr. et al. (2016), percebe-se grande o efeito do modelo no engajamento dos funcionários e na identificação organizacional, sendo uma medida que avalia a razão entre a parte explicada pelo modelo e a parte não explicada (Ringle, Bido, \& Silva, 2014). O critério de validade preditiva $\left(Q^{2}\right)$ evidencia quanto o modelo se aproxima do esperado de acordo com a sua predição, indicado por Hair Jr. et al. (2016) que os valores devem ser superiores a zero e que um modelo perfeito teria um coeficiente igual a 1 . Conforme os valores dos construtos para este critério depreendem-se que existe conformidade no modelo ajustado.

As hipóteses de pesquisa confirmaram-se conforme resultados apresentados na Tabela 4. Assim, é possível indicar que a RSC influencia positivamente no engajamento e na identificação organizacional, e este também no engajamento do funcionário.

A primeira hipótese buscou testar se existe uma relação positiva entre a RSC e o engajamento do funcionário, o que foi confirmado a um nível de significância de $1 \%(\beta=0,529, p<0,01)$. Este achado evidencia o engajamento dos funcionários como um resultado do desempenho de RSC da organização (Azim, 2016). Além disso, também está alinhado com os achados de Albdour e Altarawneh (2012), que confirmaram que a RSC é um bom preditor dos resultados do funcionário, do sucesso organizacional e do desempenho financeiro.

Apesar dos pressupostos de Carroll (1979) atribuírem às organizações a responsabilidade para com as comunidades plurais, tanto internas quanto externas, a validação do construto RSC no modelo utilizado revela um estranhamento da percepção dos funcionários quanto à prática de RSC voltada ao público externo. O constructo é composto preponderantemente com as variáveis relacionadas aos stakeholders, em geral, e aos próprios funcionários, em particular, permitindo observar distanciamento do que não pertence à sua própria comunidade. Como já mencionado na literatura, o engajamento recebe uma maior carga de comunalidades da percepção com práticas responsáveis para com seu próprio grupo, relacionadas à saúde, bem-estar, treinamento, igualdade de oportunidades e ambiente saudável de trabalho (Ferreira \& Oliveira, 2014; Turker, 2009).

Essas evidências, também observadas em outros estudos, destacam limitações quanto à efetividade da RSC externa no que tange ao engajamento do funcionário. Isso significa que se a organização planeja usar as práticas de RSC como forma de alcançar maior engajamento interno, então suas ações devem focar, principalmente, em seus próprios funcionários, que se envolverão mais na implementação de comportamentos responsáveis (Collier \& Esteban, 2007). 
A segunda hipótese, que contém o elemento fundamental da premissa teórica formulada, testou e validou que há relação positiva entre identificação organizacional e o engajamento do indivíduo. A relação foi suportada, positiva e estatisticamente significante $(\beta=0,424, p<0,01)$. Esse resultado indica que quanto maior a identificação do indivíduo com a organização, maior é o seu engajamento aplicado nas suas atividades. Ademais, este achado reforça a percepção de unidade entre a organização e o indivíduo (Ashforth \& Mael 1989; Dutton, Dukerith \& Harquail, 1994; Glavas \& Godwin, 2013; Mael \& Ashforth, 1992), e por consequência de o engajamento ser um meio para a organização alcançar o sucesso e consequentemente o do próprio indivíduo. Ao corroborar a literatura que relaciona a identificação organizacional a um comportamento (Jenkins, 2005; Tavares, 2001), a relação com o engajamento pode também ser percebida como utilitarista, visto que quanto mais o indivíduo se engaja nas suas atividades para resultar em bons resultados organizacionais (Rich et al., 2010), o sucesso da organização reflete em maior status para seus colaboradores.

Na última hipótese (H3), o interesse foi entender se a identificação organizacional atua como mediadora da RSC no engajamento dos funcionários, cujo resultado confirmou tal relação $(\beta=0,578$, p <0,01). Esse achado complementa Kahn (1990) ao argumentar que o engajamento é influenciado pelo mecanismo das condições de trabalho, entre outros. Essas evidências corroboram a proposta de Pratt (1998) de que a partir de um objetivo social, os indivíduos se percebem mais conectados à organização. Tal conexão pode transferir os objetivos da empresa para o indivíduo conquistando o seu engajamento no trabalho para um melhor desempenho organizacional (Rich et al., 2010).

Essas são evidências que reforçam a percepção de que as práticas de RSC podem incentivar o indivíduo a se identificar com a organização, como num processo de cidadania organizacional (Dutton et al., 2010; Ferreira \& Oliveira, 2014; Glavas, \& Godwin, 2013; Peterson, 2004). Com características distintas, mas ainda no mesmo sentido, os achados corroboram a indicação de Montgomery e Ramus (2008), de que os indivíduos estão dispostos a sacrificar uma parte significativa de seus salários, incluindo o sacrifício do seu esforço e intensidade na consecução dos objetivos organizacionais.

As evidências da pesquisa reforçam a literatura de que as organizações que desenvolvem maior percepção de práticas de RSC entre seus stakeholders, conseguem engajar, mais e melhor, seus funcionários nas suas atividades laborais, resultando em maior produtividade. Nesse contexto, indivíduos com maior identificação nas crenças e valores da organização maximizam esse processo de engajamento.

\section{CONCLUSÃO}

Este estudo objetivou verificar em que medida a RSC mediada pela identificação organizacional influencia no engajamento dos funcionários de um grupo de empresas localizadas na região centro sul do estado do Paraná. Para tanto confirmou-se que há relações positivas e significativas entre a percepção de RSC da empresa e o engajamento dos funcionários mediada pela identificação organizacional. Esses achados sugerem, portanto, que a RSC percebida tem o impacto mais forte nos funcionários quando eles se sentem mais identificados com a organização.

Os resultados deste estudo de caso, de que a identificação organizacional medeia a relação entre a percepção das práticas de RSC e o engajamento profissional, corroboram outros estudos identificados na literatura (Kahn, 1990; Pratt, 1998; Rich et al., 2010). Isso tem implicações importantes para a RSC e para o comportamento organizacional, pois permite refletir como os modelos (top-down ou bottom-up) podem afetar nas percepções dos indivíduos acerca da RSC. Desse modo, essas evidências contribuem ao reforçar a literatura de RSC, que construiu principalmente modelos baseados na estratégia e nas políticas de uma organização sem levar em consideração se e como a RSC está incorporada nos colaboradores além de não buscar entender as percepções destes a respeito das práticas de RSC da organização e suas consequências diretas e indiretas (Aguinis \& Glavas, 2013; Rich et al., 2010). 
A RSC deve ser incorporada nas práticas organizacionais, de tal forma que faça parte da estratégia, dos produtos, serviços e relacionamentos de uma organização. Esses aspectos reforçam a importância de conhecer as práticas de RSC das organizações e como isso é incorporado pelos funcionários em seu próprio ambiente laboral. Pesquisas futuras também podem explorar o porquê, como e quando os funcionários são afetados positiva e negativamente pela RSC, quais variáveis individuais podem ser consideradas e as percepções qualitativas.

Dentre as limitações neste estudo está a sua configuração transversal. Tal limitação pode ser superada com estudos que incluam outras avaliações, experimentais e/ou longitudinais, assim como em amostras de diferentes níveis hierárquicos (operacional, tático e estratégico).

O aumento da compreensão sobre a influência das práticas de RSC nos sentimentos, atitudes e comportamentos dos indivíduos, relacionados ao engajamento e identificação organizacional, depende da realização de mais pesquisas com essa finalidade. Esse é o sentido das recomendações de Aguinis e Glavas (2013), que enfatizam a necessidade de incluir o nível individual nas investigações sobre a RSC. A fim de contribuir com a expansão do conhecimento do tema estudado, pode-se adaptar a modelagem aqui utilizada para a realização de pesquisas comparativas entre organizações com diferentes características.

\section{REFERÊNCIAS}

Aguinis, H., \& Glavas, A. (2013). Embedded versus peripheral corporate social responsibility: Psychological foundations. Industrial and Organizational Psychology, 6(4), 314-332.

Albdour, A. A., \& Altarawneh, I. I. (2012). Corporate social responsibility and employee engagement in Jordan. International Journal of Business and Management, 7(16), 89.

Ashforth, B. E., \& Mael, F. (1989). Social identity theory and the organization. Academy of Management Review, 14(1), 20-39.

Azim, M. T. (2016). Responsabilidade Social Corporativa e comportamento do funcionário: papel mediador do compromisso organizacional. Revista Brasileira de Gestão de Negócios, 18(60), 207-225.

Bakker, A. B. (2011). An evidence-based model of work engagement. Current Directions in Psychological Science, 20(4), 265-269.

Bakker, A. B., \& Leiter, M. P. (2010). Where to go from here: Integration and future research on work engagement. Work engagement: A handbook of essential theory and research, 181-196.

Bakker, A. B., Albrecht, S. L., \& Leiter, M. P. (2011). Work engagement: Further reflections on the state of play. European journal of work and organizational psychology, 20(1), 74-88.

Baron, R. M., \& Kenny, D. A. (1986). The moderator-mediator variable distinction in social psychological research: Conceptual, strategic, and statistical considerations. Journal of personality and social psychology, 51(6), 1173.

Bhattacharya, C. B., Sen, S., \& Korschun, D. (2008). Using corporate social responsibility to win the war for talent. MIT Sloan management review, 49(2).

Bowen, H. R. (1957). Responsabilidades sociais do homem de negócios. Editora Civilização Brasileira.

Brislin, R. W. (1970). Back-translation for cross-cultural research. Journal of cross-cultural psychology, 1(3), 185-216.

Carroll, A. B. (1979). A three-dimensional conceptual model of corporate performance. Academy of management review, 4(4), 497-505.

Carroll, A. B. (1991). The pyramid of corporate social responsibility: Toward the moral management of organizational stakeholders. Business horizons, 34(4), 39-48.

Collier, J., \& Esteban, R. (2007). Corporate social responsibility and employee commitment. Business ethics: A European review, 16(1), 19-33. 
Dijkstra, T. K. (2010). Latent variables and indices: Herman Wold's basic design and partial least squares. In Handbook of partial least squares (pp. 23-46). Springer, Berlin, Heidelberg.

Dutton, J. E., Dukerich, J. M., \& Harquail, C. V. (1994). Organizational images and member identification. Administrative science quarterly, 239-263.

Dutton, J. E., Roberts, L. M., \& Bednar, J. (2010). Pathways for positive identity construction at work: Four types of positive identity and the building of social resources. Academy of Management Review, 35, 265-293.

Ferreira, P., \& Oliveira, E. R. (2014). Does corporate social responsibility impact on employee engagement? Journal of Workplace Learning, 26(3/4), 232-247.

Fornell, C., \& Larcker, D. F. (1981). Structural equation models with unobservable variables and measurement error: Algebra and statistics.

Freeman, R. E. (2010). Strategic management: A stakeholder approach. Cambridge university press.

Freguete, L. M., Nossa, V., \& Funchal, B. (2015). Responsabilidade social corporativa e desempenho financeiro das empresas brasileiras na crise de 2008. Revista de Administração Contemporânea, 19(2), 232-248.

Geisser, S. (1974). A predictive approach to the random effect model. Biometrika, 61(1), 101-107.

Glavas, A., \& Piderit, S. K. (2009). How does doing good matter. Journal of Corporate Citizenship, 36(3), 5170.

Glavas, A., \& Godwin, L. N. (2013). Is the perception of 'goodness' good enough? Exploring the relationship between perceived corporate social responsibility and employee organizational identification. Journal of business ethics, 114(1), 15-27.

Glavas, A. (2016). Corporate social responsibility and employee engagement: Enabling employees to employ more of their whole selves at work. Frontiers in psychology, 7, 796.

Hair Jr, J. F., Hult, G.T., Ringle, C. M., \& Sarsted, M. (2016). A primer on partial least squares structural equation modeling (PLS-SEM). Sage publications, 2016.

Hansen, S. D., Dunford, B. B., Boss, A. D., Boss, R. W., \& Angermeier, I. (2011). Corporate social responsibility and the benefits of employee trust: A cross-disciplinary perspective. Journal of business ethics, 102(1), 29-45.

Jenkins, S. P. (2005). Survival analysis. Unpublished manuscript, Institute for Social and Economic Research. University of Essex, Colchester, UK, 42, 54-56.

Joyner, B. E., \& Payne, D. (2002). Evolution and implementation: A study of values, business ethics and corporate social responsibility. Journal of Business Ethics, 41, 297-311.

Kahn, W. A. (1990). Psychological conditions of personal engagement and disengagement at work. Academy of management journal, 33(4), 692-724.

Lee, M. D. P. (2008). A review of the theories of corporate social responsibility: Its evolutionary path and the road ahead. International journal of management reviews, 10(1), 53-73.

MacKinnon, D. P., Lockwood, C. M., \& Williams, J. (2004). Confidence limits for the indirect effect: Distribution of the product and resampling methods. Multivariate behavioral research, 39(1), 99-128.

Mael, F., \& Ashforth, B. E. (1992). Alumni and their alma mater: A partial test of the reformulated model of organizational identification. Journal of organizational Behavior, 13(2), 103-123.

Marra, A. V., Fonseca, J. A., \& Marques, A. L. (2014). O processo de identificação organizacional ante a reforma administrativa: um estudo exploratório. RAM. Revista de Administração Mackenzie, 15(1), 4972. 
Montgomery, D. B., \& Ramus, C. A. (2008). Including corporate social responsibility, environmental sustainability, and ethics in calibrating MBA job preferences. Working Paper No. 1981, Stanford University Graduate School of Business

Oliveira, L. B. D., \& Rocha, J. D. C. (2017). Engajamento no trabalho: antecedentes individuais e situacionais e sua relação com a intenção de rotatividade. Revista Brasileira de Gestão de Negócios, 19(65), 415431.

Peterson, D. K. (2004). The relationship between perceptions of corporate citizenship and organizational commitment. Business \& society, 43(3), 296-319.

Pratt, M. G. (1998). To be or not to be: Central questions in organizational identification. In D. A. Whetten \& P. Godfrey (Eds.), Identity in organizations: Developing theory through conversations (pp. 171-207). Thousand Oaks, CA: Sage.

Rich, B. L., Lepine, J. A., \& Crawford, E. R. (2010). Job engagement: Antecedents and effects on job performance. Academy of Management Journal, 53(3), 617-635.

Ringle, C. M., Wende, S., \& Becker, J. M. (2015). SmartPLS 3. Boenningstedt: SmartPLS GmbH. http://www.smartpls.com.

Ringle, C., Silva, D., \& Bido, D. S. (2014). Modelagem de Equações Estruturais com utilização do Smartpls. Revista Brasileira de Marketing, 13(2), 54-71.

Rios, A. L. F. (2009). As relações entre satisfação, longevidade no trabalho e comprometimento organizacional em uma empresa do segmento metalúrgico de Fortaleza. Revista de Psicologia, 2(1), 49-70.

Robinson, D., Hooker, H. \& Hayday, S. (2007). Engagement: The Continuing Story, Institute for Employment Studies. London.

Saks, A. M. (2006). Antecedents and consequences of employee engagement. Journal of managerial psychology.

Serpa, D. A. F., \& Fourneau, L. F. (2007). Responsabilidade social corporativa: uma investigação sobre a percepção do consumidor. Revista de Administração Contemporânea, 11(3), 83-103.

Shuck, B., \& Wollard, K. (2010). Employee engagement and HRD: A seminal review of the foundations. Human resource development review, 9(1), 89-110.

Stone, M. (1974). Cross-validatory choice and assessment of statistical predictions. Journal of the Royal Statistical Society: Series B (Methodological), 36(2), 111-133.

Tajfel, H., Turner, J. C., Austin, W. G., \& Worchel, S. (1979). An integrative theory of intergroup conflict. Organizational identity: A reader, 56, 65.

Tavares, S. (2001). Vinculação dos indivíduos às organizações. Manual de psicossociologia das organizações, 307-333.

Tsourvakas, G., \& Yfantidou, I. (2018). Corporate social responsibility influences employee engagement. Social Responsibility Journal, 14(1), 123-137.

Turker, D. (2009). Measuring corporate social responsibility: A scale development study. Journal of business ethics, 85(4), 411-427.

Wood, D. J. (2010). Measuring corporate social performance: A review. International journal of management reviews, 12(1), 50-84.

Yin, K. (1994). Case study research: design and methods. Publications S, ed. Beverly Hills. Yousaf, H. Q., Ali, I., Sajjad, A., \& Ilyas, M. (2016). Impact of Internal Corporate Social Responsibility on Employees Engagement a Study of Moderated Mediation Model. International Journal of Sciences: Basic and Applied Research, 30(5), 226-243. 